\title{
Synthesis of Novel 2-Aryl-3-(2-morpholinoethyl)-1,3-thiazinan-4-ones Via Ultrasound Irradiation
}

\author{
Daniela P. Gouvêa, ${ }^{a}$ Gabriele A. Berwaldt, ${ }^{a}$ Patrícia D. Neuenfeldt, ${ }^{b}$ Ricardo J. Nunes, ${ }^{b}$ \\ Wanda P. Almeida ${ }^{c}$ and Wilson Cunico $*, a$
}

\author{
${ }^{a}$ Laboratório de Química Aplicada a Bioativos (LaQuiABio), Universidade Federal de Pelotas \\ (UFPel), Campus Universitário, CP 354, 96010-900 Pelotas-RS, Brazil \\ ${ }^{b}$ Laboratório Estrutura e Atividade (LEAT), Universidade Federal de Santa Catarina (UFSC), \\ Campus Trindade, 88040-970 Florianópolis-SC, Brazil
}

\author{
'Laboratório de Desenvolvimento de Fármacos e Medicamentos (LAFAME), Universidade Estadual \\ de Campinas (UNICAMP), CP 6154, 13083-970 Campinas-SP, Brazil
}

\begin{abstract}
This study describes the synthesis of fourteen thiazinanones from a multicomponent reaction of 2-morpholinoehtylamine (as primary amine), arenealdehydes (as carbonyl compound) and the mercaptopropionic acid using both conventional (thermal heating) and ultrasound methodologies. Through thermal heating methodology, the thiazinanones were obtained in 49 to $97 \%$ yields for 16 hours and through sonochemistry methodology, the reaction time was reduced for 25 minutes with yields 41 to $88 \%$. The full identification and characterization of unpublished heterocycles were achieved by proton $\left({ }^{1} \mathrm{H}\right)$ and carbon $13\left({ }^{13} \mathrm{C}\right)$ nuclear magnetic resonance (NMR) spectroscopy, mass spectrometry and infrared. Some of them were also characterized by elemental analysis.
\end{abstract}

Keywords: thiazinanone, 2-morpholinoethylamine, multicomponent reaction, sonochemistry

\section{Introduction}

The cleaner ultrasound methodology has been very effective in organic synthesis, especially in cases where the reactions take too much time. ${ }^{1}$ The effect of cavitation promotes the formation, growth and collapse of bubbles in the irradiated liquid. Consequently, the resulting high temperature and pressure inside the bubbles enhances mass transfer and a turbulent flow in the liquid and the reaction time is reduced. ${ }^{2,3}$

Multicomponent reaction is a modern and environmental friendly synthetic strategy, wherein three or more starting materials are converted into one product. This kind of reaction has attracted interest in organic synthesis due the various advantages, such as reduction in reaction times, economy of the atoms and simplification of the synthetic procedures. This approach has also been widely exploited in medicinal chemistry for obtaining bioactive compound libraries ${ }^{4}$ including heterocycles. ${ }^{5}$

Small heterocyclic moieties containing atoms of nitrogen, sulfur and oxygen have been extensively studied due to their

*e-mail: wjcunico@yahoo.com.br; wilson.cunico@ufpel.edu.br diverse biological activities. Sonochemistry has been also studied to the synthesis of heterocycles, such as thiazoles, ${ }^{6}$ thiophenes, ${ }^{7}$ thiazines ${ }^{8}$ and thiadiazines. ${ }^{9}$ Our research group and others recently published the efficient ultrasound synthesis of thiazolidinones ${ }^{10-13}$ and thiazolidinediones. ${ }^{14}$ Thiazinanones also belongs to this heterocyclic class and it is important to note that, to our knowledge, the application of sonochemistry to the synthesis of thiazinanones has not been reported in the literature.

Six-membered thiazinanones show important biological properties as antioxidant, anti-inflammatory, anti-diabetic and antimicrobial. ${ }^{15-18}$ The strategies to the synthesis of thiazinanones are quite similar to the strategies to the synthesis of analogue five-membered thiazolidinones. Thus, thiazinanones could be obtained by a multicomponent reaction between a primary amine, an aldehyde (or ketone) and the mercaptopropionic acid ${ }^{15,17,19}$ or by a reaction between thioureas and $\beta$-propylhaloacetic acid. ${ }^{18}$ Moreover, these methods can be applied in two steps, multicomponent (all reactants together in the beginning of reaction) or onepot reactions, under catalysis or not.

These observations associated to the crescent interest in multicomponent reactions encouraged us to evaluate 
the effect of ultrasound on the three component synthesis of novel thiazinanones from 2-morpholinoehtylamine, arenealdhydes and mercaptopropionic acid. For comparative purpose, the reactions were performed in the presence and in the absence of ultrasonic irradiation, i.e., by conventional method.

\section{Experimental}

\section{Material and methods}

Reagents and solvents were purchased from SigmaAldrich (St. Louis, MO, USA) and were used without additional purification. Reactions in ultrasound were carried out with a microtip probe $(3 \mathrm{~mm})$ connected to a $500 \mathrm{~W}$ Sonics Vibra-Cell ultrasonic processor (Sonics \& Materials, Inc., Newtown, CT, USA), operating at $20 \mathrm{kHz}$ (25\% of the maximum power output). Reactions were monitored by thin layer chromatography (silica gel 60 F253, ultraviolet $254 \mathrm{~nm}$ ) and gas chromatography, in a gas chromatograph (GC) GC-2010 (Shimadzu Corp., Kyoto, Japan) with a column ID of $0.25 \mathrm{~mm}$ and length of $30 \mathrm{~m}$. The analyses were carried out with a 14 psi column head pressure. The temperature range was $50{ }^{\circ} \mathrm{C}(2 \mathrm{~min})$ to $250{ }^{\circ} \mathrm{C}(10 \mathrm{~min})$ with a $16{ }^{\circ} \mathrm{C} \mathrm{min}{ }^{-1}$ rate. Temperatures of the injector and detector were 250 and $270{ }^{\circ} \mathrm{C}$, respectively. Gas chromatography with mass spectrometer (GC-MS) analyses was performed on a GC-2010 plus GCMS-QP2010 SE system AOC-20i auto injector (Shimadzu, Kyoto, Japan). Proton $\left({ }^{1} \mathrm{H}\right)$ and carbon $13\left({ }^{13} \mathrm{C}\right)$ nuclear magnetic resonance (NMR) spectra were recorded on a Bruker DRX 400 spectrometer (400.14 MHz for ${ }^{1} \mathrm{H}$ and $100.61 \mathrm{MHz}$ for ${ }^{13} \mathrm{C}$ ) or on a Bruker AVANCE 600 spectrometer $(600.17 \mathrm{MHz}$ for ${ }^{1} \mathrm{H}$ and at $150.91 \mathrm{MHz}$ for ${ }^{13} \mathrm{C}$ ) or on a Bruker $\mathrm{AC} 200 \mathrm{~F}$ spectrometer $\left({ }^{1} \mathrm{H}\right.$ at $200.13 \mathrm{MHz}$ and ${ }^{13} \mathrm{C}$ at $50.32 \mathrm{MHz}$; Billerica, $\mathrm{MA}, \mathrm{USA}$ ) in $\mathrm{CDCl}_{3}$ containing tetramethylsilane (TMS) as internal standard. The ${ }^{13} \mathrm{C}$ NMR spectrum of compound 5k was recorded on Bruker AVANCE 500 spectrometer $(125.75 \mathrm{MHz})$. The fids were processed in the MestReC 4.7.0.0 (Mestrelab Research SL, Santiago de Compostela, Spain) or in the TopSpin (Bruker, Billerica, MA, USA) softwares. Figure 1 shows the atom-numbering for thiazinanones 5a-n to the identification of NMR signals. Infrared (IR) spectra were obtained in attenuated total reflectance (ATR) on a IV Agilent absorption spectrometer, model Cary 630 FTIR (Agilent Technologies, Santa Clara, CA, USA). The elemental analyses (C, H and $\mathrm{N}$ ) were used for thiazinanones $\mathbf{5 a}, \mathbf{5 b}, \mathbf{5 c}$ and $\mathbf{5 g}$ on a 2400 Series II CHNS/O Analyser equipment (PerkinElmer, Waltham, MA, USA). Analyses were consistent within ca. 0.4 theorical values.
General procedure for the synthesis of 2-(aryl)-3-(2morpholinoethyl)-1,3-thiazinan-4-ones

Synthesis of thiazinanones $\mathbf{5 a - n}$ by thermal heating methodology

In a $100 \mathrm{~mL}$ flask with a Dean-Stark apparatus, toluene (50 mL), 2-morpholinoethylamine [4-(2-aminoethyl) morpholine] 2 (1 mmol) and corresponding arenealdehyde 1a-n $(1 \mathrm{mmol})$ were added and the reaction mixture was heated at $110^{\circ} \mathrm{C}$ for $3 \mathrm{~h}$. After this time, the mercaptopropionic acid 4 ( $2 \mathrm{mmol})$ was added and the mixture was heated for additional $16 \mathrm{~h}$. The reaction mixture was washed with saturated solution of $\mathrm{NaHCO}_{3}$ $(3 \times 30 \mathrm{~mL})$, dried with $\mathrm{MgSO}_{4}$ and concentrated in vacuo to give the expected thiazinanones. When necessary, the compounds were washed with a hot hexane to furnish the pure compounds.

Synthesis of thiazinanones $\mathbf{5 a - n}$ by ultrasound methodology

A mixture of 2-morpholinoethylamine 2 (1 mmol), arenealdehyde 1a-n $(1 \mathrm{mmol})$ and mercaptopropionic acid $4(3 \mathrm{mmol})$ in toluene $(10 \mathrm{~mL})$ was sonicated for $25 \mathrm{~min}$ in an ultrasonic probe with frequency of $20 \mathrm{kHz}$. The organic layer was washed with a saturated solution of $\mathrm{NaHCO}_{3}(3 \times 30 \mathrm{~mL})$, dried with $\mathrm{MgSO}_{4}$ and concentrated in vacuo to generate the products without purification.

\section{Selected data for the thiazinanones 5a-n}

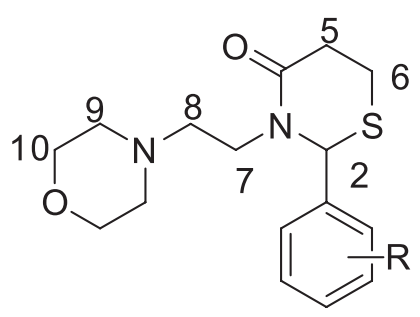

Figure 1. Atom-numbering for thiazinanones 5a-n.

3-(2-Morpholinoethyl)-2-(2-nitrophenyl)-1,3-thiazinan-4one (5a)

Yellow oil; IR (ATR) $v_{\max } / \mathrm{cm}^{-1}: 2951\left(\mathrm{C}-\mathrm{H}_{\mathrm{sp} 2}\right.$ stretch), 2866-2816 (C- $\mathrm{H}_{\mathrm{sp} 3}$ stretch), 1615 (C=O stretch), 1528-1462 (aromatic $\mathrm{C}=\mathrm{C}$ stretch), 1525 and $1357\left(\mathrm{NO}_{2}\right.$ stretch), 1121 (C-O stretch); ${ }^{1} \mathrm{H}$ NMR $\left(400 \mathrm{MHz}, \mathrm{J}_{\mathrm{H}-\mathrm{H}} \mathrm{Hz}\right) \delta 2.45-2.35$ (m, 5H, H-8b,H-9), 2.51-2.54 (m, 2H, H-5b, H-6b), 2.72-2.64 (m, 2H, H-7b, H-8a), 2.79-2.74 (m, 2H, H-5a, H-6a), 3.63 (t, 4H, J 4.6, H-10), 4.16 (dt, 1H, J 13.0, 4.2, H-7a), 6.85 (s, 1H, H-2), 7.19 (d, 1H, J 8.6, Ph-H), 7.44 (td, 1H, J 7.7, $1.2, \mathrm{Ph}-\mathrm{H}), 7.56(\mathrm{td}, 1 \mathrm{H}, J$ 7.6, 1.1, Ph-H), $8.01(\mathrm{dd}, 1 \mathrm{H}$, $J$ 8.0, 1.2, Ph-H); ${ }^{13} \mathrm{C}$ NMR (125 MHz) $\delta 21.5$ (C-6), 34.1 (C-5), 44.8 (C-7), 53.9 (2C, C-9), 56.9 (C-8), 58.3 (C-2), 
66.9 (2C, C-10), 126.9, 128.9, 132.7, 135.4, 169.9 (C-4); anal. calcd. for $\mathrm{C}_{16} \mathrm{H}_{21} \mathrm{~N}_{3} \mathrm{O}_{4} \mathrm{~S}: \mathrm{C}, 54.69 ; \mathrm{H}, 6.02 ; \mathrm{N}, 11.96$; found: C, 54.98; H, 6.40; N, 12.01; GC-MS m/z (\%): 351 $\left(\mathrm{M}^{+}, 1.5\right), 113$ (42.86), 100 (100), 56 (8).

3-(2-Morpholinoethyl)-2-(3-nitrophenyl)-1,3-thiazinan-4one $(5 b)$

White oil; IR (ATR) $v_{\max } / \mathrm{cm}^{-1}: 3062\left(\mathrm{C}-\mathrm{H}_{\mathrm{sp} 2}\right.$ stretch), 2947 and $2816\left(\mathrm{C}-\mathrm{H}_{\mathrm{sp} 3}\right.$ stretch), 1660 and 1454 (aromatic $\mathrm{C}=\mathrm{C}$ stretch $), 1525$ and $1348\left(\mathrm{NO}_{2}\right.$ stretch $), 1663(\mathrm{C}=\mathrm{O}$ stretch), 1117 (C-O stretch); ${ }^{1} \mathrm{H}$ NMR $\left(400 \mathrm{MHz}, J_{\mathrm{H}-\mathrm{H}} \mathrm{Hz}\right)$ $\delta$ 2.47-2.30 (m, 8H), 2.79-2.69 (m, 1H), 3.66-3.65 (m, 4H, H-9), 4,06-3,81 (m, 2H), 5.83 (s, 1H, H-2), 7.57 (m, 2H, Ph$\mathrm{H}), 8.13$ (q, 2H, $J$ 7.6, Ph-H); ${ }^{13} \mathrm{C}$ NMR (100 MHz) $\delta 20.1$ (C-6), 39.6 (C-5), 42.3 (C-7), 53.6 (2C, C-9), 55.7 (C-8), 61.3 (C-2), 66.8 (2C, C-10), 121.9, 123.9, 130.3, 132.7, 141.9, 148.6, 174.2 (C-4); anal. calcd. for $\mathrm{C}_{16} \mathrm{H}_{21} \mathrm{~N}_{3} \mathrm{O}_{4} \mathrm{~S}$ : C, 54.69; H, 6.02; N, 11.96; found: C, 54.40; H, 5.97; N, 11.82; GC-MS m/z (\%): $351\left(\mathrm{M}^{+}, 0.98\right), 113$ (32.21), 100 (100), 56 (9.14).

3-(2-Morpholinoethyl)-2-(4-nitrophenyl)-1,3-thiazinan-4one $(\mathbf{5 c})$

Brown oil; IR (ATR) $v_{\max } / \mathrm{cm}^{-1}: 3065\left(\mathrm{C}-\mathrm{H}_{\mathrm{sp} 2}\right.$ stretch), 2952-2812 (C- $\mathrm{H}_{\mathrm{sp} 3}$ stretch), 1596 and 1456 (aromatic $\mathrm{C}=\mathrm{C}$ stretch $), 1519$ and $1348\left(\mathrm{NO}_{2}\right.$ stretch $), 1631(\mathrm{C}=\mathrm{O}$ stretch), 1119 (C-O stretch); ${ }^{1} \mathrm{H}$ NMR $\left(400 \mathrm{MHz}, J_{\mathrm{H}-\mathrm{H}} \mathrm{Hz}\right)$ $\delta$ 2.48-2.33 (m, 5H, H-9, H-8b), 2.66-2.60 (m, 3H, H-5b, H-6b, H-8a), 2.81-2.79 (m, 3H, H-5a, H-6a, H-7a), 3.61-3.58 (m, 4H, H-10), 4.20 (dt, 1H, J 14.0, 5.6, H-7b), 5.90 (s. $1 \mathrm{H}, \mathrm{H}-2), 7.45$ (d, 1H, J 8.6, Ph-H), 8.24 (d, $2 \mathrm{H}$, $J$ 8.8, Ph-H); ${ }^{13} \mathrm{C}$ NMR (100 MHz) $\delta 21.8$ (C-6), 34.3 (C-2), 44.9 (C-7), 53.6 (2C, C-9), 56.6 (C-8), 62.0 (C-2), 67.0 (2C, C-10), 124.3, 127.3, 130.4, 147.6, 171.8 (C-4); anal. calcd. for $\mathrm{C}_{16} \mathrm{H}_{21} \mathrm{~N}_{3} \mathrm{O}_{4} \mathrm{~S}$ : C, 54.69; H, 6,02; N, 11.96; found: C, 54.42; H, 6.10; N, 11.84; GC-MS m/z (\%): 351 $\left(\mathrm{M}^{+}, 1.12\right), 113$ (13.12), 100 (100), 44 (2.02).

2-(2-Fluorophenyl)-3-(2-morpholinoethyl)-1,3-thiazinan-4one $(5 d)$

Yellow oil; IR (ATR) $v_{\max } / \mathrm{cm}^{-1}: 2959\left(\mathrm{C}-\mathrm{H}_{\mathrm{sp} 2}\right.$ stretch), 2925 and $2863\left(\mathrm{C}-\mathrm{H}_{\mathrm{sp} 3}\right.$ stretch), $1650(\mathrm{C}=\mathrm{O}$ stretch $), 1584-$ 1422 (aromatic $\mathrm{C}=\mathrm{C}$ stretch), 1116 (C-O stretch); ${ }^{1} \mathrm{H}$ NMR $\left(400 \mathrm{MHz}, J_{\mathrm{H}-\mathrm{H}} \mathrm{Hz}\right) \delta 2.44-2.34(\mathrm{~m}, 5 \mathrm{H}, \mathrm{H}-9, \mathrm{H}-8 \mathrm{~b})$, 2.60-2.54 (m, 2H, H-5b, H-6b), 2.67 (dt, 1H, J 13.9, 6.1, H-8a), 2.84-2.74 (m, 3H, H-5a, H-6a, H-7b), 3.62-3.60 (m, 4H, H-10), 4.06 (dt, 1H, J 13.8, 5.4, H-7a), 6.10 (s, 1H, H-2), 7.00 ( m, 2H, Ph-H), 7.27 (2d, 2H, J 7.2, 6.2, $\mathrm{Ph}-\mathrm{H}) ;{ }^{13} \mathrm{C}$ NMR $\left(100 \mathrm{MHz}, J_{\mathrm{C}-\mathrm{F}} \mathrm{Hz}\right) \delta 21.5(\mathrm{C}-6), 34.5$ (C-2), 44.0 (C-7), 53.7 (C-8), 56.0 (2C, C-9), 57.0 (C-2), 67.0 (2C, C-10), 116.2 (J 20.9), 126.8 (d, J 2.4), 127.0 (d,
$J$ 11.9), 129.68 (d, J 8.3), 159.5 (d, J248.5), 169.3 (C-4); GC-MS m/z (\%): $324\left(\mathrm{M}^{+}, 1.65\right), 113$ (43.12), 100 (100), 86 (5.77), 44 (1.96).

2-(3-Fluorophenyl)-3-(2-morpholinoethyl)1,3-thiazinan-4one $(5 e)$

Yellow oil; IR (ATR) $v_{\max } / \mathrm{cm}^{-1}: 2961\left(\mathrm{C}-\mathrm{H}_{\mathrm{sp} 2}\right.$ stretch), 2947 and 2929 and 2818 (C- $\mathrm{H}_{\mathrm{sp} 3}$ stretch), 1618 (C=O stretch), 1590 and 1402 (aromatic $\mathrm{C}=\mathrm{C}$ stretch), 1117 (C-O stretch); ${ }^{1} \mathrm{H}$ NMR $\left(600 \mathrm{MHz}, J_{\mathrm{H}-\mathrm{H}} \mathrm{Hz}\right) \delta 2.45-2.34(\mathrm{~m}, 5 \mathrm{H}, \mathrm{H}-8 \mathrm{~b}$, H-9), 2.60-2.53 (m, 2H, H-6b, H-8a), 2.81-2.71 (m, 4H, H-5a, H-6a, H-5b, H-7b), 3.62-3.57 (m, 4H, H-10), 4.07 (dt, $1 \mathrm{H}, J 13.9,5.1, \mathrm{H}-7 \mathrm{a}), 5.75$ (s, 1H, H-2), 6.98-6.91 (m, 3H, Ph-H), 7.28 (2d, 1H, J 7.9, 5.8, Ph-H); ${ }^{13} \mathrm{C}$ NMR (150 MHz, $\left.J_{\mathrm{C}-\mathrm{F}} \mathrm{Hz}\right) \delta 21.8(\mathrm{C}-6), 34.4(\mathrm{C}-2), 44.6(\mathrm{C}-7), 53.8(\mathrm{C}-8), 55.5$ (2C, C-9), 62.2 (C-2), 67.1 (2C, C-10), 113.7 (d, J 22.7), 115.1 ( $J$ 21.2), 122.1 ( $J$ 2.8), 130.2 ( $J$ 8.4), 142.6 ( $J$ 6.5), 162.9 (d, J 247.3), 169.2 (C-4); GC-MS m/z (\%): 324 (1.56), 113 (30.74), 100 (100), 86 (5.58), 56 (9.32).

2-(2-Chlorophenyl)-3-(2-morpholinoethyl)1,3-thiazinan-4one (5f)

White oil; IR (ATR) $v_{\max } / \mathrm{cm}^{-1}: 2951\left(\mathrm{C}-\mathrm{H}_{\mathrm{sp} 2}\right.$ stretch), 2868 and $2818\left(\mathrm{C}-\mathrm{H}_{\mathrm{sp} 3}\right.$ stretch $), 1668(\mathrm{C}=\mathrm{O}$ stretch $), 1594$ and 1409 (aromatic $\mathrm{C}=\mathrm{C}$ stretch), 1113 (C-O stretch); ${ }^{1} \mathrm{H}$ NMR $\left(200 \mathrm{MHz}, J_{\mathrm{H}-\mathrm{H}} \mathrm{Hz}\right) \delta 2.41-2.30(\mathrm{~m}, 5 \mathrm{H}, \mathrm{H}-8 \mathrm{~b}$, H-9), 2.68-2.51(m, 3H, H-5b, H-6b, H-8a), 2.78-2.70 (m, 1H, H-7b), 2.82-278 (m, 2H, H-5a, H-6a), 3.61 (t, 4H, J 4.7, H-10), 4.03-4.01 (m, 1H, H-7a), 6.16 (s, 1H, H-2), 7.01 (dt, 1H, J 4.7, 3.1, Ph-H), 7.22 (dt, 2H, J 4.6, 4.4, Ph-H), 7.39 (dt, $1 \mathrm{H}, J$ 4.6, 3.8, Ph-H); ${ }^{13} \mathrm{C}$ NMR (50 MHz) $\delta 21.1$ (C-6), 34.4 (C-5), $44.1(\mathrm{C}-7), 53.8$ (2C, C-9), 56.9 (C-8), 59.6 (C-2), 67.0 (2C, C-10), 126.5, 126.6, 129.3, 130.27, 132.6, 136.3, 169.3 (C4); GC-MS m/z (\%): $340\left(\mathrm{M}^{+}, 1.81\right)$, 113 (47.70), 100 (100), 56 (9.89).

2-(3-Chlorophenyl)-3-(2-morpholinoethyl)-1,3-thiazinan4-one $(\mathbf{5 g})$

Yellow oil; IR (ATR) $v_{\max } / \mathrm{cm}^{-1}: 3050\left(\mathrm{C}-\mathrm{H}_{\mathrm{sp} 2}\right.$ stretch), 2935-2809 (C- $\mathrm{H}_{\mathrm{sp} 3}$ stretch), 1619 (C=O stretch), 1597 and 1465 (aromatic $\mathrm{C}=\mathrm{C}$ stretch), 1109 (C-O stretch); ${ }^{1} \mathrm{H}$ NMR (400 MHz, $J_{\mathrm{H}-\mathrm{H}} \mathrm{Hz}$ ) $\delta$ 2.46-2.33 (m, 4H, H-8b, H-9), 2.612.52 ( m, 2H, H-6b, H-8a), 2.81-2.69 (m, 4H, H-5a, H-6a, H-7b, H-5b), 3.60 (q, 4H, ${ }^{3}$ J 4.7, H-10), 4.06 (dt, 1H, J 13.9, 5.2, H-7a), 5.73 (s, 1H, H-2), 7.07 (dt, 1H, J 6.6, 1.7, Ph-H), 7.23 (dt, 2H, J 7.9, 6.4, Ph-H); ${ }^{13} \mathrm{C}$ NMR (100 MHz) $\delta 169.3$ (C-4), 141.9, 134.7, 129.8, 126.6, 124.6, 67.0 (2C, C-10), 62.2 (C-2), 56.6 (C-8), 53.8 (2C, C-9), 44.6 (C-7), 34.4 (C-5), 21.8 (C-6); anal. calcd. for $\mathrm{C}_{16} \mathrm{H}_{21} \mathrm{~N}_{2} \mathrm{ClO}_{2} \mathrm{~S}$ : C, 56.38; H, 6.21; N, 8.22; found: C, 56.57; H, 6.23; N, 8.35; GC-MS $\mathrm{m} / \mathrm{z}(\%): 340\left(\mathrm{M}^{+}, 1.20\right), 113$ (32.21), 100 (100), 56 (9.14). 
2-(4-Chlorophenyl)-3-(2-morpholinoethyl)1,3-thiazinan-4one $(5 h)$

White oil; IR (ATR) $v_{\max } / \mathrm{cm}^{-1}: 2940\left(\mathrm{C}-\mathrm{H}_{\mathrm{sp} 2}\right.$ stretch), 2858-2825 (C- $\mathrm{H}_{\mathrm{sp} 3}$ stretch), 1508 ( $\mathrm{C}=\mathrm{O}$ stretch), 1576 and 1463 (aromatic $\mathrm{C}=\mathrm{C}$ stretch), 1102 (C-O stretch); ${ }^{1} \mathrm{H}$ NMR $\left(250 \mathrm{MHz}, J_{\mathrm{H}-\mathrm{H}} \mathrm{Hz}\right) \delta 2.46-2.36(\mathrm{~m}, 5 \mathrm{H}, \mathrm{H}-9, \mathrm{H}-8 \mathrm{~b})$, 2.59-2.52 (m, 2H, H-6b, H-8a), 2.79-2.68 (m. 4H, H-5a, H-5b, H-6a, H-7b), 3.61-3.59 (m, 4H, H-10), 4.08 (dt, 1H, $J$ 13.7, 5.6, H-7a), 5.75 (s, 1H, H-2), 7.13 (d, 2H, $J$ 8.0, $\mathrm{Ph}-\mathrm{H}), 7.29$ (d, 2H, J 8.3, Ph-H); ${ }^{13} \mathrm{C}$ NMR (100 MHz) $\delta 169.3(\mathrm{C}-4), 138.3,133.9,128.8,127.9,67.0$ (2C, C-10), 62.2 (C-2), 56.5 (C-8), 53.8 (2C, C-9), 44.5 (C-7), 34.5 (C-5), 21.3 (C-6); GC-MS m/z (\%): $340\left(\mathrm{M}^{+}, 1.14\right), 113$ (28.16), 100 (100), 86 (5.83), 56 (8.93).

2-(2-Methoxyphenyl)-3-(2-morpholinoethyl)-1,3-thiazinan4-one (5i)

Yellow oil; IR (ATR) $v_{\max } / \mathrm{cm}^{-1}: 2947\left(\mathrm{C}-\mathrm{H}_{\mathrm{sp} 2}\right.$ stretch), 2846-2811 (C- $\mathrm{H}_{\mathrm{sp} 3}$ stretch), 1634 (C=O stretch), 1599 and 1458 (aromatic $\mathrm{C}=\mathrm{C}$ stretch), 1110 (C-O stretch); ${ }^{1} \mathrm{H}$ NMR $\left(600 \mathrm{MHz}, J_{\mathrm{H}-\mathrm{H}} \mathrm{Hz}\right) \delta 2.42-2.34(\mathrm{~m}, 5 \mathrm{H}, \mathrm{H}-8 \mathrm{~b}, \mathrm{H}-9)$, 2.58-2.51 (m, 2H, H-6b, H-8a), 2.62 (m, 1H, J 13.9, 5.0, H-7b), 2.81-2.76 (m, 3H, H-5, H-6a), 3.62 (q, 4H, J 4.4, $\mathrm{H}-10), 3.93$ (s, 3H, $\left.\mathrm{OCH}_{3}\right), 4.03$ (dt, 1H, J 13.6, 4.7, H-7a), 6.05 (s, 1H, H-2), 6.87 (q, 2H, J 7.1, Ph-H), 6.92 (dd, 1H, $J$ 7.5, 1.7, Ph-H), 7.21 (dt, $1 \mathrm{H}, J$ 7.7, 1.8, Ph-H); ${ }^{13} \mathrm{C}$ NMR (150 MHz) $\delta 21.5$ (C-6), 34.6 (C-5), 44.2 (C-7), 53.7 (2C, C-9), 56.5 (C-8), 58.6 (C-2), 67.0 (2C, C-10), 111.0, 119.9, 126.0, 129.2, 155.9, 169.6 (C4); GC-MS m/z (\%): $336\left(\mathrm{M}^{+}\right.$, 3.71), 222 (6.20), 113 (66.26), 100 (100), 56 (11.51).

2-(3-Methoxyphenyl)-3-(2-morpholinoethyl)1,3-thiazinan4-one (5j)

Brown oil; IR (ATR) $v_{\max } / \mathrm{cm}^{-1}: 2958\left(\mathrm{C}-\mathrm{H}_{\mathrm{sp} 2}\right.$ stretch), 2852-2807 (C- $\mathrm{H}_{\mathrm{sp} 3}$ stretch), $1611(\mathrm{C}=\mathrm{O}$ stretch), 1507 and 1458 (aromatic $\mathrm{C}=\mathrm{C}$ stretch), 1111 (C-O stretch); ${ }^{1} \mathrm{H}$ NMR $\left(400 \mathrm{MHz}, J_{\mathrm{H}-\mathrm{H}} \mathrm{Hz}\right) \delta$ 2.45-2.34 (m, 5H, H-8b, H-9), 2.60-2.50 (m, 2H, H-7a, H-8a), 2.79-2.68 (m, 4H, H5, H-6a, H-7b), 3.61-3.58 (m, 4H, H-10), 3.74 (s, 3H, $\left.\mathrm{OCH}_{3}\right), 4.05$ (dt, 1H, J 13.8, 5.2, H-6b), 5.71 (s, 1H, H-2), 6.91 (dd, 2H, $J$ 8.7, 2.0, Ph-H), 7.10 (dd, 1H, J 8.6, 1.7, Ph-H); ${ }^{13} \mathrm{C} \mathrm{NMR}$ (100 MHz) $\delta 21.5$ (C-6), 34.6 (C-5), 44.2 (C-7), 53.7 (2C, $\mathrm{C}-9), 55.3\left(\mathrm{OCH}_{3}\right), 56.3(\mathrm{C}-8), 62.3$ (C-2), 66.9 (2C, C-10), 114.0, 127.8, 131.3, 159.4, 169.4 (C-4); GC-MS m/z (\%): $336\left(\mathrm{M}^{+}, 2.55\right), 222$ (1.68), 113 (42.86), 100 (100), 56 (9.69).

2-(4-Methoxyphenyl)-3-(2-morpholinoethyl)1,3-thiazinan4-one (5k)

Yellow oil; IR (ATR) $v_{\max } / \mathrm{cm}^{-1}: 2941\left(\mathrm{C}-\mathrm{H}_{\mathrm{sp} 2}\right.$ stretch), $2851\left(\mathrm{C}-\mathrm{H}_{\mathrm{sp} 3}\right.$ stretch), 1601 and 1460 (aromatic $\mathrm{C}=\mathrm{C}$ stretch), 1627 (C=O stretch), 1115 (C-O stretch); ${ }^{1} \mathrm{H}$ NMR $(400 \mathrm{MHz}$,
$\left.J_{\mathrm{H}-\mathrm{H}} \mathrm{Hz}\right) \delta 2.45-2.33(\mathrm{~m}, 5 \mathrm{H}, \mathrm{H}-9, \mathrm{H}-8 \mathrm{~b}), 2.62-2.50(\mathrm{~m}, 2 \mathrm{H}$, H-6b, H-8a), 2.78-2.71 (m, 4H, H-7b, H-5, H-6a), 3.6-3.56 (m, 4H, H-10), 3.74 (s, 3H, $\mathrm{OCH}_{3}$ ), 4.05 (dt, 1H, J 13.8, 5.3, H-7a), 5.71 (s, 1H, H-2), 6.81 (d, 2H, J 8.7, Ph-H), 7.09 (d, $2 \mathrm{H}, J$ 8.7, Ph-H); ${ }^{13} \mathrm{C}$ NMR (125 MHz) $\delta 21.8$ (C-6), 34.5 (C-5), 53.8 (2C, C-9), $55.4\left(\mathrm{OCH}_{3}\right), 56.4(\mathrm{C}-8), 62.45(\mathrm{C}-2)$, 67.0 (2C, C-10), 114.0, 127.8, 132.3, 159.4, 169.5 (C4); GC-MS m/z (\%): $336\left(\mathrm{M}^{+}, 2.14\right), 222$ (6.70), 113 (36.21), 100 (100), 86 (5.48), 44 (2.70).

2-(2-Hydroxyphenyl)-3-(2-morpholinoethyl)1,3-thiazinan4-one (5I)

Brown oil; IR (ATR) $v_{\max } / \mathrm{cm}^{-1}: 3344$ (OH stretch), $3070\left(\mathrm{C}-\mathrm{H}_{\mathrm{sp} 2}\right.$ stretch), 2950 and $2899\left(\mathrm{C}-\mathrm{H}_{\mathrm{sp} 3}\right.$ stretch $), 1615$ (C=O stretch), 1591 and 1441 (aromatic $\mathrm{C}=\mathrm{C}$ stretch), 1115 (C-O stretch); ${ }^{1} \mathrm{H}$ NMR $\left(600 \mathrm{MHz}, J_{\mathrm{H}-\mathrm{H}} \mathrm{Hz}\right) \delta 2.51-2.42$ (m, 5H, H-8b, H-9), 2.60-2.54 (m, 2H, H-6b, H-8a), 2.83-2.70 (m, 4H, H5, H-6a, H-7b), 3.62 (q, 4H, J 4.2, H-10), 4.08 (dt, 1H, J 13.8, 5.3, H-7a), 5.65 (s, 1H, H-2), 6.73 (dd, $2 \mathrm{H}, J$ 7.9, 1.8, Ph-H), 7.00 (d, 2H, J 7.9, Ph-H); ${ }^{13} \mathrm{C}$ NMR (150 MHz) $\delta 21.7$ (C-6), 34.4 (C-5), 44.4 (C-7), 62.5 (C-2), 53.6 (2C, C-9), 56.1 (C-8), 66.7 (2C, C-10), 115.5, 127.8, 129.8, 156.7, 170.0 (C4); GC-MS $m / z$ (\%): $322\left(\mathrm{M}^{+}, 0.50\right)$, 113 (43.55), 100 (100), 86 (2.17), 44 (20.68).

2-(3-Hydroxyphenyl)-3-(2-morpholinoethyl)1,3-thiazinan4-one $(5 \mathrm{~m})$

Brown oil; IR (ATR) $v_{\max } / \mathrm{cm}^{-1}: 3244$ (OH stretch), $2949\left(\mathrm{C}-\mathrm{H}_{\mathrm{sp} 2}\right.$ stretch), 2944 (OH stretch), 2861 and 2820 (C- $\mathrm{H}_{\mathrm{sp} 3}$ stretch), 1605 (C=O stretch), 1526-1456 (aromatic $\mathrm{C}=\mathrm{C}$ stretch), 1108 (C-O stretch); ${ }^{1} \mathrm{H}$ NMR $(400 \mathrm{MHz}$, $\left.J_{\mathrm{H}-\mathrm{H}} \mathrm{Hz}\right) \delta 2.50-2.38(\mathrm{~m}, 5 \mathrm{H}, \mathrm{H}-8 \mathrm{~b}, \mathrm{H}-9), 2.60-2.54$ (m, 2H, H-6b, H-8a), 2.84-2.72 (m, 4H, H-5, H-6a, H-7a), 3.61 (q, 4H, J 4.2, H10), 4.09 (dt, 1H, J 13.8, 5.3, H7b), 5.69 (s, 1H, H2), 6.62 (d, 1H, J 1.8, Ph-H), 6.71 (dd, 2H, J 7.9, $1.8, \mathrm{Ph}-\mathrm{H}), 7.24$ (d, 2H, J 7.9, Ph-H); ${ }^{13} \mathrm{C}$ NMR (100 MHz) $\delta 21.6$ (C-6), 34.5 (C-5), 44.8 (C-7), 54.7 (2C, C-9), 57.3 (C-8), 63.6 (C-2), 67.9 (2C, C-10), 114.1, 116.3, 119.5, 131.2, 141.9, 157.7, 170.9 (C4); GC-MS $m / z(\%): 322\left(\mathrm{M}^{+}\right.$, 1.23), 113 (35.67), 100 (100), 86 (6.93), 44 (2.00).

2-(4-Hydroxyphenyl)-3-(2-morpholinoethyl)1,3-thiazinan4-one (5n)

Yellow oil; IR (ATR) $v_{\max } / \mathrm{cm}^{-1}: 3305$ (OH stretch), $3056\left(\mathrm{C}-\mathrm{H}_{\mathrm{sp} 2}\right.$ stretch $), 2970$ and $2869\left(\mathrm{C}-\mathrm{H}_{\mathrm{sp} 3}\right.$ stretch $), 1636$ $(\mathrm{C}=\mathrm{O}$ stretch), 1610 and 1461 (aromatic $\mathrm{C}=\mathrm{C}$ stretch), 1112 (C-O stretch); ${ }^{1} \mathrm{H}$ NMR $\left(600 \mathrm{MHz}, J_{\mathrm{H}-\mathrm{H}} \mathrm{Hz}\right) \delta 2.50-2.42$ (m, $5 \mathrm{H}, \mathrm{H}-8 \mathrm{~b}, \mathrm{H}-9), 2.60-2.54$ (m, 2H, H-6b, H-8a), 2.84-2.70 (m, 4H, H-5, H-6a, H-7b), 3.44 (dt, 1H, J 13.3, 5.7, H-7a), 3.71 (q, H4, J 4.6, H-10), 4.16 (dt, 1H, J 13.7, 5.6, H-6b), $5.74(\mathrm{~s}, 1 \mathrm{H}, \mathrm{H}-2), 6.82(\mathrm{~d}, 2 \mathrm{H}, J$ 8.6, Ph-H), $7.08(\mathrm{~d}, 2 \mathrm{H}$, 
$J$ 8.5, Ph-H); ${ }^{13} \mathrm{C}$ NMR (150 MHz) $\delta 21.7$ (C-6), 34.5 (C-5), 44.4 (C-7), 53.7 (2C, C-9), 56.2 (C-8), 62.5 (C-2), 66.7 (2C, C-10), 115.6, 127.9, 156.8, 170.1 (C-4); GC-MS $m / z$ (\%): $322\left(\mathrm{M}^{+}, 1.36\right), 113$ (32.58), 100 (100), 86 (7.14), 44 (3.09).

\section{Results and Discussion}

One-pot synthesis of thiazinanones by the conventional thermal heating method

To the synthesis of thiazinanones $\mathbf{5 a - n}$, three components were used as the starting materials: the mercaptoacetic acid, arenealdehydes bearing electron-withdrawn or electronreleasing groups, and the 2-morpholinoethylamine. The choice for 2-morpholinoethylamine as amine core was due the biological importance of heterocyclic morpholine. Compounds with this scaffold feature a wide range of biological activities, recently reviewed by Pal'chkov. ${ }^{20}$

Our first purpose was the synthesis of heterocycles by the conventional thermal heating methodology (Scheme 1). The expected compounds were synthesized through the one-pot reaction procedure in toluene refluxing. In this procedure, the intermediate imine was completely formed ( $3 \mathrm{~h}$ ) before the cyclocondensation step to the thiazinanones $(16 \mathrm{~h})$. The water was removed by azeotropic distillation using a Dean-Stark apparatus and the progresses of all reactions were monitored by thin layer chromatography (TLC) and/or by GC. The heterocycles were obtained in moderate to excellent yields after the purification by washing with hot hexane/ethyl acetate (9:1).
Sonification effect on the multicomponent synthesis of thiazinanones

The six-membered thiazinanone has structural similarity to the five-membered thiazolidinone, so the reaction condition using ultrasound was based in such heterocycle, according to a recent work published by us. ${ }^{12}$ 2-Morpholinoethylamine is an aliphatic amine and the first experiment was carried out in a multicomponent reaction of amine 2, 2-nitrobenzaldehyde 1a and mercaptopropionic acid 4 ( 3 equivalents) in toluene for $5 \mathrm{~min}$. However, it was observed the starting material aldehyde in TLC and GC. The next experiment was monitored by TLC in ten, fifteen, twenty and twenty-five minutes. The complete formation of thiazinanone $\mathbf{5 a}$ (up to $99 \%$ in GC) was observed in $25 \mathrm{~min}$. The product was isolated and all spectrometric and spectroscopic data are identical to those for thiazinanone 5a prepared by the conventional method. Further, the proportion of the mercaptopropionic acid $\mathbf{4}$ was also studied and no significant difference on yields was found when 1 , 2 or 3 equivalents of acid were used.

Found the best condition, the synthesis of all thiazinanones 5a-n were carried out in a mixture of $1 \mathrm{mmol}$ of arenaldehydes 1a-n, $1 \mathrm{mmol}$ of amine $\mathbf{2}$ and $1 \mathrm{mmol}$ of the mercaptopropionic acid $\mathbf{4}$ that was sonicated for $25 \mathrm{~min}$ in toluene (Scheme 2). The heterocycles 5a-n were obtained in a range of $41-88 \%$, without further purification.

The comparative yields of thiazinanones 5a-n are summarized in Table 1. The electronic nature of arenealdehyde substituent did not affect the yields<smiles>NCCN1CCOCC1</smiles>

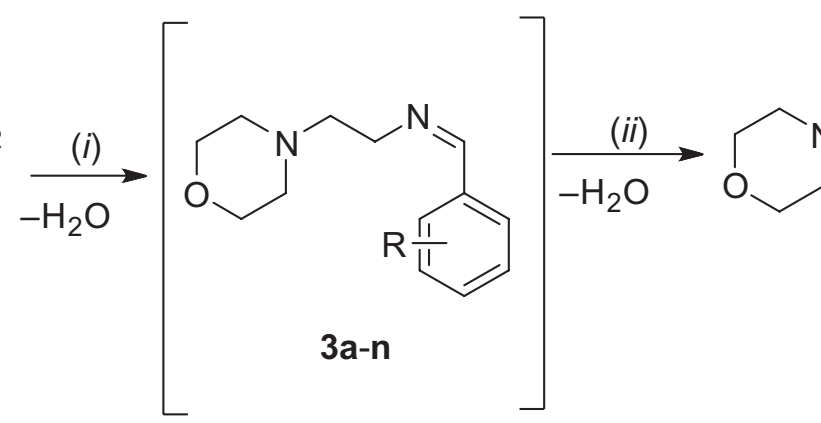<smiles>[R1]c1cccc(C2SCCC(=O)N2CCN2CCOCC2)c1</smiles>

5a-n

Scheme 1. (i) Toluene, $110{ }^{\circ} \mathrm{C}, 3 \mathrm{~h}$; (ii) $\mathrm{HSCH}_{2} \mathrm{CH}_{2} \mathrm{COOH}, 110{ }^{\circ} \mathrm{C}, 16 \mathrm{~h}(49-97 \%)$.<smiles>O=C([18F])c1[R]cccc1</smiles>

1a-n<smiles>NCCN1CCOCC1</smiles>

2<smiles>O=C1CCSC(c2c[R17]ccc2)N1CCN1CCOCC1</smiles>

5a-n 
of products as well the position, with exception for thiazinanones 51-n bearing a phenolic group that showed lower yields. A possible explanation is the polar hydroxyl group that increase water solubility and an amount of product might be lost in the extraction procedure.

As we expected, the sonochemisty methodology afford the pure thiazinanones in lower reaction times than conventional heating (25 minutes to 19 hours) in quite similar yields. Other important advantage of ultrasound methodology is the absence of water remove procedure, e.g., azeotropic distillation, desiccant agents.

Table 1. Yields of thiazinanones 5a-n synthesized by ultrasound and thermal heating methodologies

\begin{tabular}{lccc}
\hline \multirow{2}{*}{ entry } & $\mathrm{R}$ & \multicolumn{2}{c}{ Yield $^{\mathrm{a}} \%$} \\
\cline { 3 - 4 } & & Thermal heating & Ultrasound \\
\hline $\mathbf{5 a}$ & $2-\mathrm{NO}_{2}$ & 87 & 71 \\
$\mathbf{5 b}$ & $3-\mathrm{NO}_{2}$ & 79 & 86 \\
$\mathbf{5 c}$ & $4-\mathrm{NO}_{2}$ & 89 & 73 \\
$\mathbf{5 d}$ & $2-\mathrm{F}$ & 97 & 82 \\
$\mathbf{5 e}$ & $3-\mathrm{F}$ & 74 & 48 \\
$\mathbf{5 f}$ & $2-\mathrm{Cl}$ & 85 & 46 \\
$\mathbf{5 g}$ & $3-\mathrm{Cl}$ & 91 & 86 \\
$\mathbf{5 h}$ & $4-\mathrm{Cl}$ & 64 & 78 \\
$\mathbf{5 i}$ & $2-\mathrm{OCH}_{3}$ & 88 & 85 \\
$\mathbf{5 j}$ & $3-\mathrm{OCH} \mathrm{H}_{3}$ & 87 & 75 \\
$\mathbf{5 k}$ & $4-\mathrm{OCH} \mathrm{H}_{3}$ & 84 & 88 \\
$\mathbf{5 l}$ & $2-\mathrm{OH}$ & 60 & 53 \\
$\mathbf{5 m}$ & $3-\mathrm{OH}$ & 72 & 55 \\
$\mathbf{5 n}$ & $4-\mathrm{OH}$ & 49 & 41 \\
\hline
\end{tabular}

${ }^{\text {a }}$ Yield of isolated compounds.

The fourteen unpublished thiazinanones were identified and characterized by mass spectrometry and ${ }^{1} \mathrm{H}$ and ${ }^{13} \mathrm{C}$ NMR spectroscopy. The 2D NMR techniques heteronuclear multiple quantum correlation (HMQC) and heteronuclear multiple bond correlation (HMBC) were necessary to the correct assignments of hydrogens and carbons. The ${ }^{1} \mathrm{H}$ NMR spectrum shows that the aromatic signals appear in the range of 8.24-7.00 ppm, the $\mathrm{H} 2$ hydrogen as a singlet at $6.85-5.68 \mathrm{ppm}$. The H10 and $\mathrm{H} 9$ of the morpholine ring are assigned as a triplet or multiplet at 3.63-3.57 ppm and as a multiplet in the range of 2.48-2.33 ppm, respectively. The $\mathrm{H} 7 \mathrm{a}$ of ethylene bridge is shown as a double triplet at $4.20-4.06 \mathrm{ppm}$ $\left({ }^{2} J=14.0-13.0 \mathrm{~Hz},{ }^{3} J=5.6-4.2 \mathrm{~Hz}\right)$ and $\mathrm{H} 7 \mathrm{~b}$ and $\mathrm{H} 8$ appear as multiplets with superposition of signals. The four diastereotopic $\mathrm{H} 5$ and $\mathrm{H} 6$ of thiazinanone ring are shown as multiplets due the superposition of signals in the range of 2.82-2.51 ppm. In ${ }^{13} \mathrm{C}$ NMR, the $\mathrm{C} 10$ and C9 of morpholine ring appear at 66.7-67.9 ppm and 53.6-56.0 ppm, respectively. The chemical shift of ethylene carbons shows that $\mathrm{C} 8$ is deshilded at $67.9-66.7 \mathrm{ppm}$ and C7 are shielded at 56.0-53.6 ppm, according to HMQC spectra. The carbons of thiazinanones are assigned as 174.2-169.2 ppm for the carbonyl (C4), 62.2-57.0 ppm for the $\mathrm{C} 2,34.6-34.1 \mathrm{ppm}$ for $\mathrm{C} 5$ and 21.8-21.5 ppm for C6. The HMBC confirm the assignment of C5 and C6.

\section{Conclusions}

Therefore, the sonochemistry was efficient as an attractive strategy to promote the synthesis of thiazinanones through a multicomponent reaction. The products were obtained in good yields in shorter reaction times $(25 \mathrm{~min})$ when compared to thermal heating methodology (19 h). In addition, no water removal was necessary when the ultrasound was used.

\section{Suplemantary Information}

Supplementary information with analytical data (mass, ${ }^{1} \mathrm{H}$ and ${ }^{13} \mathrm{C}$ NMR and infrared spectra) is available free of charge at http://jbcs.sbq.org.br as PDF file.

\section{Acknowledgments}

Authors thank UFPel and FAPERGS (Process 11/2068-7) for the financial support. W. P. A. is also grateful to FAPESP (Process 2013/18203-5) and W. C. is grateful to CNPq (Process 307330/2012-5).

\section{References}

1. Coelho, F.; Almeida, W.; Veronese, D.; Mateus, C. R.; Lopes, E. C. S.; Rossi, R. C.; Silveira, G. P. C.; Pavam, C. H.; Tetrahedron 2002, 58, 7437.

2. Cella, R.; Stefani, H. A.; Tetrahedron 2009, 65, 2619.

3. Cravotto, G.; Cintas, P.; Chem. Soc. Rev. 2006, 35, 180.

4. Song, Y.; Dong, Y. F.; Yang, W. F.; Yang, G. L.; Ultrason. Sonochem. 2015, 22, 119.

5. Fascio, M. L.; Errea, M. I.; D’Accorso, N. B.; Eur. J. Med. Chem. 2015, 90, 666.

6. Gomba, S. M.; Khalil, K. D.; Molecules 2012, 17, 9335.

7. Liang, C.; Wang, D. L. X.; Zhang, Q.; Yao, Q.; J. Sulfur Chem. 2013, 34, 458.

8. Arya, K.; Rawat, D. S.; Sasai, H.; Green Chem. 2012, 14, 1956.

9. Kumar, S. K.; Rambabu, D.; Kumar, C. H. V.; Sreenivas, B. Y.; Prasad, K. R. S.; Rao, M. V. B.; Pal, M.; RSC Adv. 2013, 3, 24863. 
10. Nikalje, A. P. G.; Shaikh, S. I.; Muley, A.; Khan, F. A. K.; Sangshetti, J. N.; Shaikh, S.; Arch. Pharm. (Weinheim, Ger.) 2014, 347, 756.

11. Mamaghani, M.; Loghmanifar, A.; Taati, M. R.; Ultrason. Sonochem. 2011, 18, 45.

12. Gouvêa, D. P.; Bareño, V. D. O.; Bosenbecker, J.; Drawanz, B. B.; Neuenfeldt, P. D.; Siqueira, G. M.; Cunico, W.; Ultrason. Sonochem. 2012, 19, 1127.

13. Neuenfeldt, P. D.; Drawanz, B. B.; Aguiar, A. C. C.; Figueiredo, F.; Krettli, A.; Cunico, W.; Synthesis 2011, 23, 3866.

14. Drawanz, B. B.; Ribeiro, C. S.; Masteloto, H. G.; Neuenfeldt, P. D.; Pereira, C. M. P.; Siqueira, G. M.; Cunico, W.; Ultrason. Sonochem. 2014, 21, 1615.

15. Bosenbecker, J.; Bareño, V. D. O.; Difabio, R.; Vasconcellos, F. A.; Dutra, F. S. P.; Oliveira, P. S.; Barschak, A. G.; Stefanello, F. M.; Cunico, W.; J. Biochem. Mol. Toxicol. 2014, 28, 425.
16. Zebardast, T.; Zarghi, A.; Daraie, B.; Hedayati, M.; Dadrass, O. G.; Bioorg. Med. Chem. Lett. 2009, 19, 3162.

17. Raza, S.; Srivastava, S. P.; Srivastava, D. S.; Srivastava, A. K.; Haq, W.; Katti, S. B.; Eur. J. Med. Chem. 2013, 63, 611.

18. Mohamed, K. S.; Abdelhamid, A. A.; Omara, W.; Jaber, A. A.; Albayati, M.; J. Chem. Pharm. Res. 2013, 5, 19.

19. Zhou, H.; Liu, A.; Li, X.; Ma, X.; Feng, W.; Zhang, W.; Yan, B.; J. Comb. Chem. 2008, 10, 303.

20. Pal'chkov, V. A.; Russ. J. Org. Chem. 2013, 49, 787.

Submitted: November 10, 2015 Published online: January 19, 2016

FAPERGS/CAPES and FAPESP have sponsored the publication of this article. 